\title{
Avaliação técnica e econômica da adubação nitrogenada em Urochloa brizantha cv. Marandu na Região do Vale do Acre, Brasil
}

Technical and economic evaluation of nitrogen fertilization in Urochloa brizantha cv. Marandu in the Acre Valley Region, Brazil

Evaluación técnica y económica de fertilización nitrogenada en Urochloa brizantha cv. Marandu en la región de Vale do Acre, Brasil

Eduardo Mitke Brandão Reis ORCID: https://orcid.org/0000-0001-8513-4398 Universidade Federal do Acre, Brasil E-mail: edumitke@hotmail.com

Ítalo Câmara de Almeida

ORCID: https://orcid.org/0000-0002-3349-1796 Universidade Federal do Espírito Santo, Brasil E-mail: almeidaicvet@gmail.com

Vinícius Borges Taquetti

ORCID: https://orcid.org/0000-0001-6067-1664 Universidade Federal do Espírito Santo, Brasi E-mail: viniciustaquetti@hotmail.com

Nayara Maria Pedrosa Praça ORCID: https://orcid.org/0000-0001-7931-5707 Universidade Federal do Espírito Santo, Brasil E-mail: nay.pedrosa@gmail.com

Felipe Berbari Neto

ORCID: https://orcid.org/0000-0001-8367-0314 Universidade Federal do Espírito Santo, Brasil E-mail: berbarineto@hotmail.com

\begin{abstract}
Resumo
Objetivou-se avaliar o desempenho técnico e econômico da adubação nitrogenada de Uroclhoa brizantha cv. Marandu na região do vale do Acre, entre os meses de agosto de 2011 a junho de 2012. Os tratamentos consistiram de sete doses de nitrogênio $(0 ; 33,75 ; 67,50 ; 101,25 ; 135,00 ; 168,75$ e 202,50kg de N/ha/ano), correspondendo a $0 ; 75 ; 150 ; 225 ; 300$; 375 e $450 \mathrm{~kg}$ ureia/ha respectivamente. As aplicações foram realizadas a lanço, distribuídas em três etapas. Com o acréscimo das dosagens de nitrogênio observou-se aumento não linear na matéria seca, proteína bruta, cinzas e extrato etéreo nas amostras analisadas, que assim, apresentaram bons parâmetros de valor nutritivo e de desenvolvimento. A dosagem de nitrogênio que obteve melhor desempenho em relação ao custo da adubação nitrogenada foi a de $67,50 \mathrm{~kg}$ de N/ha, pois este apresentou os melhores custos de produção (U\$49,86 de MS e U\$30,36 de PB) tornando-se a opção mais vantajosa economicamente. A dosagem de nitrogênio que apresentou melhor desempenho agronômico foi de $168,75 \mathrm{Kg} \mathrm{N} / \mathrm{ha}$, apresentando valor de proteína bruta de $17,78 \%$. Os parâmetros de valor nutritivo e de desenvolvimento da forrageira tropical $U$. brizantha cv. Marandu utilizada na região do vale do Acre apresentaram desempenho agronômico satisfatório.
\end{abstract}

Palavras-chave: Amazônia; Custo; Nitrogênio; Pastagem.

\begin{abstract}
The objective of this study was to evaluate the technical and economic performance of Uroclhoa brizantha cv. Marandu in the Acre Valley region, from August 2011 to June 2012. The treatments consisted of seven nitrogen doses $(0 ; 33.75$; $67.50 ; 101.25 ; 135.00 ; 168.75$ and $202.50 \mathrm{~kg} \mathrm{~N} / \mathrm{ha} /$ year), corresponding to $0 ; 75 ; 150 ; 225 ; 300 ; 375$ and $450 \mathrm{~kg}$ urea/ha respectively. The applications were carried out at haul, distributed in three stages. With the increase of nitrogen dosages, nonlinear increase in dry matter, crude protein, ash and ether extract was observed in the analyzed samples, which presented good parameters of nutritional value and development. The nitrogen dosage that obtained the best performance in relation to the cost of nitrogen fertilization was $67.50 \mathrm{~kg} \mathrm{~N} / \mathrm{ha}$, as it presented the best production costs (U\$ 49.86 DM and U\$ $30.36 \mathrm{CP}$ ) becoming the most economically advantageous option. The nitrogen dosage that presented the best agronomic performance was $168.75 \mathrm{Kg} \mathrm{N} / \mathrm{ha}$, with crude protein value of $17.78 \%$. The nutritional and developmental parameters of tropical forage $U$. brizantha cv. Marandu used in the Acre Valley region showed satisfactory agronomic performance.
\end{abstract}

Keywords: Amazon; Cost; Nitrogen; Pasture. 


\section{Resumen}

El objetivo fue evaluar el desempeño técnico y económico de la fertilización nitrogenada de Uroclhoa brizantha cv. Marandu en la región del Valle de Acre, de agosto de 2011 a junio de 2012. Los tratamientos consistieron en siete tasas de nitrógeno $(0 ; 33.75 ; 67.50 ; 101.25 ; 135.00 ; 168.75$ y $202.50 \mathrm{~kg}$ de N/ha/año), correspondientes a $0 ; 75 ; 150 ; 225$; $300 ; 375$ y $450 \mathrm{~kg}$ de urea/ha respectivamente. Las aplicaciones se llevaron a cabo por difusión, distribuidas en tres etapas. Con la adición de las dosis de nitrógeno se observó un aumento no lineal de materia seca, proteína cruda, cenizas y extracto etéreo en las muestras analizadas, las cuales presentaron así buenos parámetros de valor nutritivo y desarrollo. La dosis de nitrógeno que tuvo el mejor desempeño en relación al costo de la fertilización nitrogenada fue de $67.50 \mathrm{~kg}$ de N/ha, ya que ésta tuvo los mejores costos de producción (U\$ 49.86 de MS y U\$30.36 de CP) por lo que es la opción más ventajosa económicamente. La dosis de nitrógeno que presentó el mejor comportamiento agronómico fue de 168.75 $\mathrm{kg} \mathrm{N} / \mathrm{ha}$, con un valor de proteína bruta de $17.78 \%$. El valor nutricional y los parámetros de desarrollo del forraje tropical U. brizantha cv. Marandu utilizado en la región del Valle de Acre mostró un desempeño agronómico satisfactorio.

Palabras clave: Amazon; Costo; Nitrógeno; Pastar.

\section{Introdução}

As pastagens são a forma mais prática e econômica para alimentação de animais especializados na produção de leite e carne, constituindo a base de sustentação da pecuária no Brasil (Tonello et al., 2011; Poppi et al., 2018). As áreas de pastagens no Brasil ocupam cerca de 170 milhões de hectares, no entanto, até 70\% das pastagens cultivadas apresentam algum grau de degradação, sendo a degradação o maior entrave para a sustentabilidade da produção animal (Dias-Filho, 2011; Gosch et al., 2021).

As limitações na produção de forragem com valor nutritivo adequado estão associadas à baixa fertilidade do solo e ao manejo inadequado das pastagens. A utilização da adubação é essencial para manter esse sistema produtivo. Nesse sentido, o nitrogênio, é importante para o crescimento e produção das plantas. Uma vez que há aumento nos níveis de nitrogênio no solo, há também maiores taxas de desenvolvimento e crescimento da planta, e consequentemente, aumenta o acúmulo de forragem por área cultivada (Duru \& Ducroq, 2000; Momesso et al., 2019).

A adubação nitrogenada favorece a taxa de crescimento cultural diretamente por meio da rápida restauração do índice de área foliar. Desta forma, este nutriente é fundamental para a manutenção da produtividade das pastagens e para a sua sustentabilidade, uma vez que a deficiência de nitrogênio é o primeiro fator desencadeador do processo de degradação. Além disso, a adubação nitrogenada pode melhorar a qualidade da forrgaem aumentando seu teor de proteína bruta (Alves et al., 2008; Kumar et al., 2021).

Dentre as espécies forrageiras mais cultivadas no Brasil, destaca-se a Uroclhoa brizantha cv. Marandu. Na região Norte, estima-se que estejam ocupadas com essa gramínea cerca de $65 \%$ do território. Nas últimas décadas, $U$. brizantha tem sido introduzida no Acre para formação de pastagens e utilização na pecuária bovina (Machado et al., 2010; Boddey et al., 2020).

Considerando a escassez de literatura quando se trata da região Amazônica, objetivou-se avaliar qual o volume ideal de adubo nitrogenado a ser empregado para maximizar o desempenho agronômico, nutritivo e econômico da $U$. brizantha cv. Marandu nas condições climáticas da mesorregião do Vale do Acre.

\section{Metodologia}

O trabalho foi conduzido entre agosto de 2011 a junho de 2012 na área de experimentação em forragicultura da

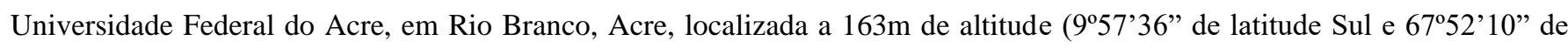
longitude Oeste). A região avaliada apresenta umidade relativa do ar elevada, temperatura máxima local de aproximadamente $32^{\circ} \mathrm{C}$ e temperatura média anual de $25^{\circ} \mathrm{C}$.

Antecedendo a semeadura de $U$. brizantha cv. Marandu, foi realizada análise química do solo, a partir de amostras retiradas à profundidade de zero a $0,40 \mathrm{~m}$ (Tabela 1), de acordo com metodologia de Raij et al. (2001). Após análise, foi realizada calagem e adubação. A área de pastagem foi dividida em 56 parcelas de $4 \mathrm{~m} 2$ (sete tratamentos com oito repetições), sendo a 
mesma semeada com a forrageira Uroclhoa brizantha cv. Marandu. A semeadura foi feita a lanço, realizada em março de 2011, na quantidade de $20 \mathrm{~kg}$ de sementes por hectare, com profundidade de $2 \mathrm{~cm}$. O valor cultural (VC) das sementes de U. brizantha cv. Marandu foi de $40 \%$ de germinação. O preparo da área experimental constou de aração e gradagem leve. Imediatamente após a distribuição das sementes foi realizada outra gradagem leve, com o uso de grade niveladora de arrasto.

Todas as correções de solo foram realizadas baseadas na análise previamente coletada. A saturação de base foi elevada para 60\% utilizando calcário dolomítico com $85 \%$ de poder relativo de neutralização total (PRNT). Os índices de fósforo (P) foram elevados através da aplicação do fertilizante superfosfato simples para que atingisse o valor mínimo de $10 \mathrm{mmolc} / \mathrm{dm} 3$. As correções dos índices de potássio $(\mathrm{K})$ foram elevados através da aplicação do fertilizante cloreto de potássio $(\mathrm{KCl})$ para que atingisse os valores mínimos de 2,5\% de K na capacidade de troca de cátion (CTC em mmolc/dm3). Houve a utilização de 50 $\mathrm{Kg} / \mathrm{ha}$ de micronutrientes. A dose e o turno de irrigação foram estabelecidos com base nos totais pluviométricos anuais (1.877 a $1.982 \mathrm{~mm})$ e na temperatura média anual $\left(25^{\circ} \mathrm{C}\right)$ da região.

Os tratamentos consistiram de sete doses de nitrogênio $(0 ; 33.75 ; 67.50 ; 101.25 ; 135.00 ; 168.75$ e $202.50 \mathrm{~kg}$ de $\mathrm{N} / \mathrm{ha} /$ ano), correspondendo a $0 ; 75 ; 150 ; 225 ; 300 ; 375$ e $450 \mathrm{~kg}$ ureia/ha. As aplicações foram realizadas a lanço, distribuídas em três etapas. Nestes locais também foi realizada adubação pós-plantio com base na análise de solo. Subsequentemente ao corte de uniformização, a cada 20 dias, as parcelas foram submetidas a três cortes para a avaliação da taxa de produção de matéria seca (MS). Posteriormente a cada corte, foi realizada adubação nitrogenada.

Amostras de matéria verde foram retiradas com cutelo, mimetizando preensão animal a $5 \mathrm{~cm}$ do solo. A área amostral compreendeu um quadrado de $100 \mathrm{~cm}$ de lado $\left(1 \mathrm{~m}^{2}\right)$, disposto no centro de cada unidade experimental. Após o corte, as amostras de forragem foram acondicionadas em sacos plásticos, pesada em balança eletrônica de precisão seguida da tabulação de produção de matéria verde (PMV). Em seguida, duas amostras de 300g foram acondicionadas em sacos de papel para conservar a umidade. Posteriormente, foram utilizados $150 \mathrm{~g}$ do material coletado para a separação de lâminas e colmos com bainhas. A quantidade restante foi pesada, acondicionada em sacos de papel e seca em estufa com circulação de ar forçado por 72 horas a $60^{\circ} \mathrm{C}$ para a determinação da fitomassa seca (Van Soest, 1994). As análises de matéria seca (MS), proteína bruta (PB), extrato etéreo (EE) e cinzas (CZ) foram realizadas segundo metodologia de Silva (1990).

Para avaliação do custo econômico realizou-se o custo da adubação nitrogenada das diferentes doses de nitrogênio aplicadas em cada tratamento. Este foi analisado conforme a porcentagem de proteína que foi incluída em cada dosagem, comparando com o tempo gasto, o custo e o incremento de proteína nos diferentes tratamentos. A formação da área plantada não foi computada, pois o custo de formação dos canteiros experimentais foi o mesmo para todos os tratamentos, variando apenas, a aplicação de nitrogênio.

Para avaliação econômica, foram considerados os preços praticados na região na ocasião da realização da pesquisa. Levou-se em consideração o valor da adubação nitrogenada e o tempo de aplicação para cada tratamento, no intuito de calcular o custo total da adubação nitrogenada. $\mathrm{O}$ valor do quilo de ureia no Acre foi de U\$1,18 à época do estudo e a mão de obra baseado no salário mínimo da época (Decreto 7655/2011), de U\$ 1,38/hora.

A avaliação do custo da adubação nitrogenada foi realizada através da comparação entre os percentuais de PB e MS com custo total da adubação nitrogenada, com intuito de avaliar o tratamento com melhor desempenho econômico. Foi realizado cálculo de custo para produzir 1\% de MS e para se produzir 1\% de PB. Avaliou-se também o tratamento que obteve maior desempenho agronômico, ou seja, a dosagem de nitrogênio que resultou em maior aumento na concentração de proteína.

A distribuição dos dados coletados foi verificada através do teste de Shapiro-Wilk. Foi então empregado o teste não paramétrico de Kruskal-Wallis para comparação hierárquica entre as médias dos tratamentos de cada análise bromatológica. Toda análise estatística foi conduzida utilizando o programa Statistix® (2009), com nível de significância de 5\%. 


\section{Resultados}

A análise química do solo (Tabela 1), foi realizada antes da aplicação da adubação nitrogenada na área experimental. A partir desses dados foi realizada a calagem e a adubação de correção.

Tabela 1. Análise química do solo da estação experimental de forragicultura da Universidade Federal do Acre na camada 0-40 $\mathrm{cm}$.

\begin{tabular}{cccccccccc}
\hline \multirow{2}{*}{$\begin{array}{c}\text { P Resina } \\
\left(\mathrm{mg} / \mathrm{dm}^{3}\right)\end{array}$} & $\begin{array}{c}\mathrm{MO} \\
\left(\mathrm{g} / \mathrm{dm}^{3}\right)\end{array}$ & $\mathrm{pH}$ & $\mathrm{K}$ & $\mathrm{Ca}$ & $\mathrm{Mg}$ & $\mathrm{H}+\mathrm{Al}$ & $\mathrm{Al}$ & $\mathrm{SB}$ & $\mathrm{CTC}$ \\
\cline { 3 - 10 } & 14 & 3,9 & 0,8 & 20 & 5 & 51 & 50 & 25,8 & 76,8 \\
\hline 4 & 14 & & & & & & & & \\
\hline
\end{tabular}

Fonte: Autores.

Os resultados obtidos para a produtividade de matéria seca (MS), teores de proteína bruta (PB), extrato etéreo (EE), e cinzas (CZ) dos três cortes de Uroclhoa brizantha cv. Marandu realizados, revelou que a dose de $168.75 \mathrm{Kg} / \mathrm{N} / \mathrm{ha} / \mathrm{ano}$ apresentou melhor desempenho agronômico, ou seja, maior incremento de PB. Apenas a dose de $33.75 \mathrm{Kg} / \mathrm{N} / \mathrm{ha} / \mathrm{ano}$ não apresentou bom desempenho, pois obteve valores de PB abaixo do grupo controle. Os demais tratamentos apresentaram resposta eficiente em relação às outras amostras, considerando as quantidades de nitrogênio aplicadas à cada tratamento (Tabela 2).

Tabela 2. Valores médios de matéria seca, cinzas, extrato etéreo e proteína segundo a análise bromatológica dos sete tratamentos.

\begin{tabular}{ccccc}
\hline $\begin{array}{c}\text { Doses } \\
(\mathrm{Kg} / \mathrm{N} / \mathrm{ha} / \mathrm{ano})\end{array}$ & $\begin{array}{c}\text { Matéria Seca } \\
(\%)\end{array}$ & $\begin{array}{c}\text { Cinzas } \\
(\%)\end{array}$ & $\begin{array}{c}\text { Extrato etéreo } \\
(\%)\end{array}$ & $\begin{array}{c}\text { Proteína bruta } \\
(\%)\end{array}$ \\
\hline 0 & $24.10^{\mathrm{a}}$ & $6.80^{\mathrm{c}}$ & $1.00^{\mathrm{b}}$ & $10.99^{\mathrm{c}}$ \\
33.75 & $22.88^{\mathrm{b}}$ & $7.87^{\mathrm{abc}}$ & $1.07^{\mathrm{b}}$ & $9.82^{\mathrm{c}}$ \\
67.50 & $21.65^{\mathrm{b}}$ & $7.44^{\mathrm{bc}}$ & $1.54^{\mathrm{ab}}$ & $16.78^{\mathrm{ab}}$ \\
101.25 & $20.56^{\mathrm{b}}$ & $6.49^{\mathrm{c}}$ & $1.08^{\mathrm{b}}$ & $14.09^{\mathrm{bc}}$ \\
135.00 & $20.04^{\mathrm{ab}}$ & $8.89^{\mathrm{ab}}$ & $1.20^{\mathrm{ab}}$ & $13.09^{\mathrm{bc}}$ \\
168.75 & $21.05^{\mathrm{b}}$ & $9.13^{\mathrm{a}}$ & $1.76^{\mathrm{a}}$ & $17.78^{\mathrm{a}}$ \\
202.50 & $20.63^{\mathrm{ab}}$ & $8.70^{\mathrm{ab}}$ & $1.70^{\mathrm{a}}$ & $16.33^{\mathrm{ab}}$ \\
\hline
\end{tabular}

Valores seguidos por letras diferentes na mesma coluna diferem entre si a nível de 5\% significância. Fonte: Autores.

Em relação ao custo da adubação, o grupo controle teve custo zero, uma vez que não houve aplicação de adubação nitrogenada. A partir das doses seguintes houve aplicação de $75 \mathrm{~kg}$ de ureia por hectare por tratamento, ocasionando o custo computado do fertilizante e da mão de obra (Tabela 3).

Tabela 3. Tempo de aplicação, custo do quilo da ureia e custo total da adubação nitrogenada por parcela.

\begin{tabular}{|c|c|c|c|c|}
\hline $\begin{array}{c}\text { Doses } \\
(\mathrm{Kg} / \mathrm{N} / \mathrm{ha} / \mathrm{ano}) \\
\end{array}$ & $\begin{array}{l}\text { Tempo de } \\
\text { aplicação }\end{array}$ & $\begin{array}{l}\text { Custo do tempo de } \\
\text { aplicação (U\$) }\end{array}$ & $\begin{array}{c}\text { Custo da ureia } \\
\text { (U\$/Kg) }\end{array}$ & $\begin{array}{c}\text { Custo total por } \\
\text { parcela (U\$) }\end{array}$ \\
\hline 0 & 0.00 & 0.00 & 0.00 & 0.00 \\
\hline 33.75 & $1^{\prime} 49^{\prime \prime}$ & 0.034 & 88.50 & 88.53 \\
\hline 67.50 & $1^{\prime} 55^{\prime \prime}$ & 0.035 & 177.00 & 177.03 \\
\hline 101.25 & $2^{\prime} 01^{\prime \prime}$ & 0.046 & 265.50 & 265.54 \\
\hline 135.00 & $2^{\prime} 06^{\prime \prime}$ & 0.047 & 354.00 & 354.04 \\
\hline 168.75 & $2^{\prime} 17^{\prime \prime}$ & 0.049 & 442.50 & 442.54 \\
\hline 202.50 & $2^{\prime} 22^{\prime \prime}$ & 0.051 & 531.00 & 531.05 \\
\hline
\end{tabular}

Fonte: Autores.

De acordo com os resultados encontrados, o melhor desempenho em relação ao custo da adubação nitrogenada foi de $67.50 \mathrm{Kg} / \mathrm{N} / \mathrm{ha} / \mathrm{ano}$, pois este apresentou os melhores custos de produção (U\$30.36 de PB) tornando-se a opção mais vantajosa economicamente (Tabela 4). 
Tabela 4. Comparação entre os percentuais de proteína bruta e matéria seca com o custo total da adubação nitrogenada em diferentes dosagens de nitrogênio por aplicação.

\begin{tabular}{ccccc}
\hline Dose (Kg/N/ha/ano) & Proteína Bruta (\%) & $\begin{array}{c}\text { Diferença de PB em } \\
\text { relação Controle } \\
\text { (sem adubação) }\end{array}$ & $\begin{array}{c}\text { Custo adubação } \\
\text { nitrogenada (U\$) }\end{array}$ & $\begin{array}{c}\text { Custo de 1\% PB } \\
\text { (U\$) }\end{array}$ \\
\hline 0 & 10.96 & 0 & 0 & 0 \\
33.75 & 9.85 & -1.11 & 88.53 & 79.76 \\
67.50 & 16.79 & 5.83 & 177.03 & 30.36 \\
101.25 & 14.06 & 3.10 & 265.54 & 85.66 \\
135.00 & 13.05 & 2.09 & 354.04 & 169.40 \\
168.75 & 17.78 & 6.82 & 442.54 & 64.89 \\
202.50 & 16.33 & 5.37 & 531.05 & 98.89 \\
\hline
\end{tabular}

Fonte: Autores.

\section{Discussão}

Os valores encontrados diferem dos teores médios de PB encontrados para a U. brizantha cv. Marandu por Reis et al. (2013), variando de 8.37 a 9.79\%. Os autores afirmam que, pelo fato do parcelamento de nitrogênio apresentar maior efeito na distribuição de matéria seca, este pode promover melhor distribuição da qualidade da forrageira principalmente em relação ao teor de PB.

Teixeira et al. (2018) verificaram o efeito de diferentes doses nitrogênio (0, 100, 200 e $300 \mathrm{~kg} \mathrm{~N} / \mathrm{ha})$ e fósforo (0, 100, 200 e $300 \mathrm{~kg}$ P/ha) na produção de MS de $U$. brizantha cv. Marandu. Os autores observaram que a produção de matéria seca e verde foi influenciada pela adubação e que houve interação entre as doses de nitrogênio e fósforo, com resposta linear crescente independente da dose aplicada de fósforo.

De modo geral, os teores de PB (Tabela 2) aumentaram de acordo com as doses de nitrogênio aplicadas. Ruggieri et al. (1995), ao avaliarem doses de adubação nitrogenada e regimes de corte em Uroclhoa brizantha, verificaram que com 28 dias de intervalo de corte, os valores de PB foram de 13.50; 16.30 e 17.30\% para as doses de nitrogênio de 0, 33 e 66kg N/ha, respectivamente. Esses autores observaram que a tendência geral foi de aumento no teor de PB das folhas com acréscimo da dose de nitrogênio utilizada. Quando comparadas as doses de 0 e $100 \mathrm{~kg}$ de N/ha, verificou-se incremento médio de 22,5\% de PB nas folhas. Entretanto, quando avaliadas as demais doses sem o controle, verificou-se variação de 8 a $17 \%$ de PB (Magalhães, 2007).

As análises dos dados obtidos indicam que as doses de nitrogênio empregadas não proporcionaram aumento linear nos teores de PB, diferente do encontrado por outros autores (Nakamura et al., 2005; Teixeira et al., 2018). Isto pode estar relacionado ao fato de os 56 canteiros experimentais terem sido submetidos a escolha aleatória, fazendo com que alguns deles fossem submetidos a menor incidência solar durante o período da tarde.

Em relação a interação entre as doses de nitrogênio e cortes, observa-se que os teores de PB foram influenciados pelo aumento das doses administradas (Maranhão et al., 2009; Avelino et al., 2019). À medida em que se aumentaram estas doses, ocorreu acréscimo considerável no teor médio de PB, para o primeiro e terceiro corte. As médias dos teores de PB variaram de $10.65 \%$, para o tratamento testemunha (sem adubação nitrogenada), a 17.67\%, na maior dose de nitrogênio aplicada (202.50 kg N/ha). Tais resultados são semelhantes aos encontrados outros autores (Benneti et al., 2008; Faria et al., 2018).

Comparando o efeito das doses de nitrogênio com os resultados obtidos por outros autores, com relação a produção de forragem, a produção de MS não aumentou de acordo com as dosagens (Tabela 2). Diferente do presente estudo, Costa et al. (2009) observaram maiores índices de MS com maiores doses de nitrogênio. Porto (2017) observou efeito de interação entre diferentes cultivares de Uroclhoa spp. submetidos à adubação nitrogenada. Costa et al. (2020a) e Costa et al. (2020b), trabalhando com Uroclhoa brizantha cv. Piatã e Uroclhoa Ruziziensis, encontraram aumento linear de matéria seca verde (MSV) em doses crescentes de nitrogênio, atingindo o pico de MSV com $120 \mathrm{Kg} / \mathrm{N} / \mathrm{ha}$ e $180 \mathrm{Kg} / \mathrm{N} / \mathrm{ha}$, respectivamente. Fagundes et al. (2005), 
obtiveram crescimento de forma linear, indicando que o suprimento de nitrogênio do solo não atende à demanda das gramíneas estudadas. Embora o efeito do nitrogênio sobre o incremento de produção seja relevante, é preciso considerar que a produção de forragem não constitui a única característica para avaliar o potencial de uma forrageira (Sória, 2002; Pullanagari et al., 2018).

Os valores médios de MS variaram de $64.10 \%$ a $70.88 \%$, sendo que a dosagem de $33,75 \mathrm{Kg} / \mathrm{N} / \mathrm{ha} / \mathrm{ano}$ foi a que apresentou maior percentagem de matéria seca $(70,88 \%)$. Os tratamentos que receberam $135.00 \mathrm{Kg} / \mathrm{N} / \mathrm{ha} /$ ano e 202.50 $\mathrm{Kg} / \mathrm{N} / \mathrm{ha} /$ ano não apresentaram diferenças significativas entre si, assim como as doses de $33.75 ; 67.5 ; 101.25 ; 168.75$ $\mathrm{Kg} / \mathrm{N} / \mathrm{ha} /$ ano. Em pastagens de gramíneas tropicais pode ser obtido valor médio de $26 \mathrm{~kg}$ MS por quilo de nitrogênio podendose atingir valores de até $83 \mathrm{~kg}$ MS por quilo de nitrogênio aplicado (Martha Júnior et al., 2004).

Era esperado que o efeito do nitrogênio na produção de matéria seca fosse mais pronunciado em relação aos teores de proteína, evidenciando assim o efeito diluição, especialmente quando são aplicadas doses baixas do fertilizante nitrogenado. Quando se empregaram doses mais elevadas, teores maiores de proteína poderiam ser esperados, indicando maior acúmulo de proteína em comparação ao acúmulo de MS (Viana et al., 2011; Delevatti et al., 2019).

Quanto aos valores de cinzas, que indicam a quantidade de matéria orgânica presente na forragem, não foram obtidos resultados lineares. Assim, o tratamento que apresentou maior percentagem de cinzas foi $168.75 \mathrm{~kg} \mathrm{~N} / \mathrm{ha} / \mathrm{ano}$. Chizzotti et al. (2005) encontraram em silagem de U. brizantha $1.27 \%$ de cinzas. As oito parcelas que receberam dose de $67.50 \mathrm{~kg} \mathrm{~N} / \mathrm{ha} / \mathrm{ano}$ apresentaram melhor desempenho econômico (Tabela 2), levando-se em consideração os valores apresentados de PB (16.79\%).

Para se produzir $1 \%$ de $\mathrm{PB}$, foi observado que a dose de $33.75 \mathrm{Kg} / \mathrm{N} / \mathrm{ha} / \mathrm{ano}$ apresentou elevado custo de adubação nitrogenada. Desta forma, este tratamento não seria recomendado para aplicação, pois a produção de PB foi inferior a encontrada no grupo controle, o qual não recebeu adubação nitrogenada. Essa resposta está aquém da esperada para $U$. brizantha para as doses de nitrogênio empregadas. Tal fato poderia ser decorrente de perdas de ureia por volatilização.

A dose de $168.75 \mathrm{Kg} / \mathrm{N} / \mathrm{ha} /$ ano apresentou melhor desempenho agronômico, proporcionando melhor valor de $\mathrm{PB}$ (17.78\%). Entretanto, a dosagem de $67.50 \mathrm{Kg} / \mathrm{N} / \mathrm{ha} /$ ano se torna mais viável economicamente, pois apresentou teores de $\mathrm{PB}$ semelhante a $168.75 \mathrm{Kg} / \mathrm{N} / \mathrm{ha} /$ ano.

\section{Conclusão}

Os parâmetros de valor nutritivo e de desenvolvimento da forrageira tropical $U$. brizantha cv. Marandu utilizada na região do Vale do Acre apresentaram desempenho agronômico satisfatório.

Não se obteve aumentos lineares em relação aos teores de proteína bruta e os tratamentos com doses de nitrogênio.

A dosagem de nitrogênio que obteve o melhor desempenho em relação ao custo da adubação nitrogenada foi a de 67.50 $\mathrm{kg}$ de N/ha.

Recomenda-se a avaliação do desempenho animal, sob condições de criação extensiva contendo forrageira tropical de U. brizantha cv. Marandu, a fim de ratificar os resultados encontrados no presente estudo.

\section{Referências}

Alves, J. S., Pires, A. J. V., Matsumoto, S. N., Figueiredo, M. P. \& Ribeiro, G. S. (2008) Características morfológicas e estruturais da Brachiaria decumbens Stapf. submetidas a diferentes doses de nitrogênio e volumes de água. Acta Veterinaria Brasilica, 2 (1), 1-10.

Avelino, A. C. D., de Faria, D. A., Penso, S., Lima, D. D. O. S., Rodrigues, R. C., de Abreu, J. G., Cabral, L. S. \& Peixoto, W. M. (2019). Agronomic and bromatological traits of Brachiaria brizantha cv. Piatã as affected by nitrogen rates and cutting heights. Journal of Experimental Agriculture International, 36 , $1-11$.

Boddey, R. M., Casagrande, D. R., Homem, B. G. \& Alves, B. J. (2020). Forage legumes in grass pastures in tropical Brazil and likely impacts on greenhouse gas emissions: A review. Grass and Forage Science, 75(4), 357-371.

Brasil. (2011) Ministério do Trabalho. Decreto No 7.655, de 23 de dezembro de 2011. <http://www.normaslegais.com.br/legislacao/decreto-7655-2011.htm>. 
Chizzotti, F. H. M., Pereira, O. G., Valadares Filho, S. D. C., Garcia, R., Chizzotti, M. L., Leão, M. I. \& Pereira, D. H. (2005). Consumo, digestibilidade total e desempenho de novilhos Nelore recebendo dietas contendo diferentes proporções de silagens de Brachiaria brizantha cv. Marandu e de sorgo. Revista Brasileira de Zootecnia, 34, 2427-2436.

Costa, K. A. P., Faquin, V., Oliveira, I. P., Severiano, E. C., Simon, G. A. \& Carrijo, M. S. (2009). Extração de nutrientes pela fitomassa do capim-marandu sob doses e fontes de nitrogênio. Revista Brasileira de Saúde e Produção Animal, 10(4).

Costa, N. L., Magalhães, J. A., Bendahan, A. B., Rodrigues, A. N. A., Rodrigues, A. N. A. \& Santos, F. J. S. (2020b). Produtividade de forragem e morfogênese de Brachiaria Ruziziensis sob níveis de nitrogênio. Research, Society and Development, 9(1), 1-14.

Costa, N. L., Magalhães, J. A., Bendahan, A. B., Rodrigues, A. N. A., Rodrigues, A. N. A. \& Santos, F. J. S. (2020a). Response of Brachiaria brizantha cv. Piatã pastures to nitrogen fertilization. Research, Society and Development, 9(3), 1-14.

Delevatti, L. M., Cardoso, A. S., Barbero, R. P., Leite, R. G., Romanzini, E. P., Ruggieri, A. C. \& Reis, R. A. (2019). Effect of nitrogen application rate on yield, forage quality, and animal performance in a tropical pasture. Scientific reports, 9(1), 1-9.

Dias-Filho, M. B. (2011). Degradação de Pastagens: processo, causas e estratégias de recuperação. (4a ed.), MBDF, 204p.

Duru, M., Ducrocq, H. (2000). Growth and senescence of the successive leaves on a Cocksfoot tiller. Ontogenic development and effect of temperature. Annals of Botany, 85, 635-643.

Fagundes, J. L., Fonseca, D. M. D., Gomide, J. A., Nascimento Junior, D. D., Vitor, C. M. T., Morais, R. V. D., Mistura, C.; Reis, G. C. \& Martuscello, J. A. (2005). Acúmulo de forragem em pastos de Brachiaria decumbens adubados com nitrogênio. Pesquisa Agropecuária Brasileira, 40, $397-403$.

Faria, B. M., Morenz, M. J. F., Paciullo, D. S. C., Lopes, F. C. F. \& Gomide, C. A. D. M. (2018). Growth and bromatological characteristics of Brachiaria decumbens and Brachiaria ruziziensis under shading and nitrogen1. Revista Ciência Agronômica, 49, 529-536.

Gosch, M. S., Parente, L. L., dos Santos, C. O., Mesquita, V. V. \& Ferreira, L. G. (2021). Landsat-based assessment of the quantitative and qualitative dynamics of the pasture areas in rural settlements in the Cerrado biome, Brazil. Applied Geography, 136, 102585.

Kumar, P., Singh, G., Singh, P. D., Singh, T., Singh, A. \& Lakra, K. (2021). Effect of different crop establishment methods and nitrogen levels on the performance of kharif season rice (Oryza sativa) in the Indo-Gangatic plains of eastern Uttar Pradesh. Crop Research, 56, 1-7.

Machado, L. A., Lempp, B., Valle, C. B., Jank, L., Batista, L. A. R., Postiglioni, S. R. \& de Andrade, C. M. S. (2010). Principais espécies forrageiras utilizadas em pastagens para gado de corte. Embrapa Pecuária Sudeste.

Magalhães, A. F., Pires, A. J. V., Carvalho, G. G. P. D., Silva, F. F. D., Sousa, R. S. \& Veloso, C. M. (2007). Influência do nitrogênio e do fósforo na produção do capim-braquiária. Revista Brasileira de Zootecnia, 36, 1240-1246.

Maranhão, C. M. A., da Silva, C. C. F., Bonomo, P. \& Pires, A. J. V. (2009). Produção e composição químico-bromatológica de duas cultivares de braquiária adubadas com nitrogênio e sua relação com o índice SPAD. Acta Scientiarum. Animal Sciences, 31(2), 117-122.

Martha Júnior, G. B., Vilela, L., Barioni, L. G., Sousa, D. D. \& Barcellos, A. D. O. (2004). Manejo da adubação nitrogenada em pastagens. Simpósio sobre manejo da pastagem, 21, 155-216.

Momesso, L., Crusciol, C. A., Soratto, R. P., Vyn, T. J., Tanaka, K. S., Costa, C. H., Neto, J. F. \& Cantarella, H. (2019). Impacts of Nitrogen Management on No-Till Maize Production Following Forage Cover Crops. Agronomy Journal, 111(2), 639-649.

Nakamura, T., Miranda, C. H., Ohwaki, Y., Valéio, J. R.; Kim, Y. \& Macedo, M. C. (2005). Characterization of nitrogen utilization by Brachiaria grasses in Brazilian Savannas (Cerrados). Soil Science \& Plant Nutrition, 51(7), 973-979.

Poppi, D. P., Quigley, S. P., Silva, T. A. C. \& McLennan, S. R. (2018). Challenges of beef cattle production from tropical pastures. Revista Brasileira de Zootecnia, 47, 1-9.

Porto, E. M. V. (2017). Produção de biomassa de três cultivares do gênero Brachiaria spp. submetidos à adubação nitrogenada. Agropecuária Científica no Semiárido, 13(1), 9-14.

Pullanagari, R. R., Kereszturi, G. \& Yule, I. (2018). Integrating airborne hyperspectral, topographic, and soil data for estimating pasture quality using recursive feature elimination with random forest regression. Remote Sensing, 10(7), 1117.

Raij, B. V. \& Quaggio, J. A. (2001). Determinação de fósforo, cálcio, magnésio e potássio extraídos com resina trocadora de íons. B. van Raij, JC, andrade, H. Cantarella and JA Quaggio (Ed.), Análise química para avaliação da fertilidade de solos tropicais, 189-199.

Reis, G. L., Neto, J. V. E., de Lemos Filho, J. P., Borges, I. \& Longo, R. M. (2013). Produção e composição bromatológica do capim-marandu sob diferentes percentuais de sombreamento e doses de nitrogênio. Bioscience Journal, 29.

Ribeiro, K. G. \& Pereira, O. G. (2010) Valor nutritivo do capim-tifton 85 sob doses de nitrogênio e idades de rebrotação. Revista Brasileira de Veterinária e Zootecnia, 17(12), 560-567.

Ruggieri, A. C., Favoretto, V. \& Malheiros, E. B. (1995). Efeito de níveis de nitrogênio e regimes de corte na distribuição, na composição bromatológica e na digestibilidade in vitro da matéria seca da Brachiaria brizantha (HOCHST) Stapf. Cv. Marandu. Revista Brasileira de Zootecnia, 24(1), 21-30.

Silva, D. J. (1990). Análise de alimentos (métodos químicos e biológicos). (2a ed.), Universidade Federal de Viçosa.

Sória, L. G. T. (2002) Produtividade do capim-tanzânia (Panicum maximum Jacq. cv. Tanzânia) em função da lâmina de irrigação e de adubação nitrogenada. 182 f. Tese Doutorado. 
Research, Society and Development, v. 11, n. 1, e44411125314, 2022

(CC BY 4.0) | ISSN 2525-3409 | DOI: http://dx.doi.org/10.33448/rsd-v11i1.25314

Teixeira, R. N. V., Pereira, C. E., Kikuti, H. \& Deminicis, B. B. (2018). Brachiaria brizantha (Syn. Uroclhoa brizantha) cv. Marandu under different doses of nitrogen and phosphorus in Humaitá-AM, Brazil. Applied Research \& Agrotechnology, 11(2), 35-41.

Van Soest, P. J. (1994). Nutritional ecology of the ruminant. Cornell university press.

Viana, M. C. M., Freire, F. M., Ferreira, J. J., Macêdo, G. A. R., Cantarutti, R. B. \& Mascarenhas, M. H. T. (2011). Adubação nitrogenada na produção e composição química do capim braquiária sob pastejo rotacionado. Revista Brasileira de Zootecnia, 40, 1497-1503.

Tonello, C. L., Branco, A. F., Tsutsumi, C. Y., Ribeiro, L. B., Coneglian, S. M. \& Castañeda, R. D. (2011). Suplementação e desempenho de bovinos de corte em pastagens: tipo de forragem. Acta Scientiarum. Animal Sciences, 33(2), 199-205. 